\title{
Conversão de Termos, Homotopia, e Estrutura de Grupóide
}

\author{
Arthur F. Ramos ${ }^{1}$, Ruy J. G. B. de Queiroz ${ }^{2}$, Anjolina G. de Oliveira ${ }^{2}$ \\ ${ }^{1}$ Microsoft Redmond, USA \\ arfreita@microsoft.com \\ ${ }^{2}$ Centro de Informática, Univ. Federal de Pernambuco, Recife, Brazil \\ \{ruy, ago\}@cin.ufpe.br
}

\begin{abstract}
A relação de conversão entre termos dada por Church, que determina a igualdade entre termos, diz que dois $\lambda$-termos são o mesmo se forem conversíveis um ao outro. Em outras palavras, um caminho entre dois termos, o que sugere a possibilidade de uma semântica com base em homotopia.
\end{abstract}

\section{Introdução}

Uma ampla frente de pesquisa nos fundamentos da matemática tem sido explorada desde 2005 na tentativa de construir uma ponte entre a teoria de tipos e a teoria da homotopia, principalmente através da estrutura de grupóide revelada no contramodelo de HofmannStreicher [Hofmann and Streicher 1994] ao princípio da Unicidade de Provas de Identidade (UIP). Daí surge a homotopy type theory em que o tipo 'identidade' assume um protagonismo revelador ao possibilitar a interpretação de tipos como espaços ao invés de simplesmente conjuntos. Isso tem aberto caminho para, nas palavras de S. Awodey, "uma nova e surpreendente conexão entre Geometria, Álgebra, e Lógica, que tem vindo à tona recentemente na forma de uma interpretação da teoria construtiva de tipos de Per MartinLöf na teoria da homotopia, resultando em novos exemplos de certas estruturas algébricas que são importantes em topologia" [Awodey 2012].

Por trás dessa ampla conexão está um conceito que aparece de formas distintas em diversas áreas da matemática: caminho. A saber: (i) topologia algébrica: teoria da homotopia; (ii) categorias de ordem superior: policategorias; (iii) álgebra de ordem superior: $\infty$ groupóides; (v) lógica: provas de igualdade; e, através do $\lambda$-cálculo: (vi) sequências de reescrita. No que concerne a (v) e (vi), vale lembrar que, nas diferentes formulações da teoria de tipos (intensional ou extensional), o julgamento da forma ' $a=b: A$ ' é lido como ' $a$ e $b$ são elementos (definicionalmente) iguais do tipo $A$ '. Não há a introdução de contrapartida formal do caminho que levaria de $a$ até $b$ por meio das regras de reescrita. Em nossa abordagem acrescentamos identificadores para a composição de caminhos, de forma que o julgamento ficaria algo como ' $a={ }_{s} b: A$ ' cuja leitura seria ' $a$ é igual a $b \mathrm{em}$ razão de $s$ ' (' $s$ ' sendo a razão para reescrita), ' $s$ ' aparecendo como um termo denotando uma composição de identificadores de igualdades definicionais $(\beta, \eta, \mu, \nu$, $\xi$, etc.). Em outras palavras, ' $s$ ' é o caminho computacional (explícito) de $a$ para $b$.

\section{Fundamentos da matemática}

Como observou Vladimir Voevodsky em sua palestra no WoLLIC 2011 [?],nos últimos anos, conexões profundas e um tanto inesperadas têm sido descobertas entre as teorias construtivas de tipos e a teoria da homotopia. Tais conexões abrem caminho para que 
se construam novos fundamentos da matemática alternativamente à teoria dos conjuntos de Zermelo-Fraenkel (ZFC). Tais fundamentos se mostram bastante promissores no que diz respeito à resolução de diversos problemas em áreas não diretamente relacionadas, tais como fornecer o devido suporte para argumentos categóricos de ordem mais alta diretamente no nível da linguagem, permitir que as formalizações da matemática se tornem muito mais concisas e muito melhor adaptadas ao uso de sistemas de prova semiautomatizados (Ex. Coq, Lean), além de propiciar um meio uniforme de abordar tanto a matemática clássica quanto a matemática construtiva. Em palestra em Göteborg, Voevodsky argumentava que o principal problema prático da formalização da matemática baseada em ZFC pode ser chamado de "o problema da equivalência". Se, por um lado, a matemática em nível de conjuntos lida apenas com propriedades de conjuntos com estruturas que são invariantes sob isomorfismos, a matemática de ordem superior que manuseia categorias e seus análogos de ordem superior trata essencialmente de propriedades invariantes sob equivalências de ordem superior apropriadamente definidas. Daí, ZFC não parece dar margem a uma abordagem natural para a distinção entre construções e propriedades que respeitam equivalências daquelas que não as respeitam. ${ }^{1}$

Por outro lado, Michael Shulman em seu recente Homotopy type theory - A high-level language for invariant mathematics chama a atenção para a importância de se identificar os princípios que fundamentam as noções de equivalência em matemática: "Homotopy type theory is a high-level abstract framework for working with sameness", daí a relação de mesmice (do inglês, sameness) seria de tal forma que: (i) dois grupos são o mesmo se forem isomorfos; (ii) dois espaços topológicos são o mesmo se forem homeomorfos; (iii) duas categorias são a mesma se forem equivalentes; (iv) dois conjuntos são o mesmo se tiverem a mesma extensão (v) dois mapeamentos são homotopicamente o mesmo se um pode ser continuamente deformado para o outro.

Observando a natureza da relação de conversão entre termos dada por Church, faz sentido acrescentar à lista anterior: (vi) dois $\lambda$-termos são o mesmo se forem conversíveis um ao outro.

Conforme a definição de igualdade de termos no $\lambda$-cálculo:

Definição 2.1 ([Hindley and Seldin 2008]) $P$ é $\beta$-igual ou $\beta$-conversível a $Q$ (notação $P={ }_{\beta} Q$ ) sse $Q$ for obtido de $P$ por uma série (talvez vazia) de $\beta$-contrações e $\beta$ contrações reversas e trocas de variáveis ligadas. Ou seja, $P={ }_{\beta} Q$ sse existe $P_{0}, \ldots, P_{n}$ $(n \geq 0)$ tais que $P_{0} \equiv P, P_{n} \equiv Q,(\forall i \leq n-1)\left(P_{i} \triangleright_{1 \beta} P_{i+1}\right.$ ou $P_{i+1} \triangleright_{1 \beta} P_{i}$ or $\left.P_{i} \equiv_{\alpha} P_{i+1}\right)$.

A igualdade vem com uma força existencial. Além disso, trata-se de uma relação obtida tomando-se o fecho reflexivo, simétrico e transitivo da relação de contração em 1-passo, o que se revela com as propriedades de caminho.

Combinando as idéias de Steve Awodey sobre a interpretação dos chamados "tipos-

\footnotetext{
1 "One of the keystones of contemporary mathematics is the isomorphism invariance principle: for any statement $P$ about $X$ and any isomorphism $X \stackrel{\phi}{\simeq} X^{\prime}$, there is a statement $P_{\phi}$ about $X^{\prime}$ such that $P$ holds iff $P_{\phi}$ holds. The equality problem in formalizations comes in part from the fact that when one encodes $X$ and $X^{\prime}$ the isomorphism is lost. There is more to the equality problem than isomorphism invariance: (i) equality is a good notion for 'elements' - individuals, but fails for collections; (ii) isomorphism is a good notion for collections, but fails for collections of collections. This leads to a theory of iterated $n$-equivalences which are the correct replacements for such "iterated collection."(Foundations of Mathematics and Homotopy Theory, IAS (2006))
} 
identidade" da teoria de tipos de Per Martin-Löf como "espaços de caminhos" e suas próprias idéias sobre a interpretação dos universos dessa mesma teoria, Voevodsky construiu, em 2009, o chamado "modelo univalente"do cálculo de construções indutivas de Thierry Coquand. Tal modelo propicia uma semântica para o cálculo das construções indutivas que permite se fazer exatamente o que era necessário para se formalizar a nova abordagem aos fundamentos da matemática supracitada.

Segundo Voevodsky, a abordagem denominada de Fundamentos Univalentes, diferentemente das abordagens baseadas em ZFC e em teoria das categorias, é um sistema completo de fundamentos, ainda que muito diferente de ZFC. Para efeito de comparação, vamos admitir que quaisquer sistemas de fundamentos para a matemática, adequados tanto para o raciocínio humano quanto para a verificação através do computador, devem ter os três seguintes componentes: o primeiro componente é um sistema de dedução formal, ou seja, uma linguagem e um conjunto de regras de manipulação de sentenças nessa linguagem que sejam puramente formais, tais que o passo-a-passo dessas manipulações possa ser verificado por um programa de computador; o segundo componente é uma estrutura que forneça significado às sentenças dessa linguagem em termos de objetos mentais intuitivamente compreensíveis por seres humanos; o terceiro componente é uma estrutura que permita que seres humanos codifiquem suas ideias matemáticas em termos dos objetos diretamente associados com a linguagem.

Isso faz parte de um projeto em andamento [Ramos et al. 2017, Martínez-Rivillas and de Queiroz 2021] que, ao mesmo tempo em que busca o uso de estruturas de homotopia como grupóides no estudo da semântica da computação, também busca demonstrar a utilidade e o impacto da chamada interpretação de CurryHoward da dedução lógica na prática real de uma importante área da matemática, a saber, a teoria da homotopia. A breve citação do "Prêmio Rolf Schock 2020 em lógica e filosofia da Real Academia Sueca de Ciências"diz que foi concedido a Per Martin-Löf (compartilhado com Dag Prawitz) "pela criação da teoria construtiva dos tipos". Em declaração mais longa, o comitê do prêmio lembra que a teoria dos tipos construtiva é "uma linguagem formal em que é possível expressar a matemática construtiva"(...) "[que] também funciona como uma linguagem de programação poderosa e teve um impacto enorme em lógica, ciência da computação e, recentemente, matemática."Ao introduzir um arcabouço no qual a formalização da noção lógica de igualdade é feita por meio do chamado "tipo identidade", é apresentada a possibilidade de uma ligação surpreendente entre a reescrita de termos e conceitos geométricos como caminho e homotopia. E, de fato, a teoria de tipos de Martin-Löf (MLTT) permite fazer pontes úteis entre a teoria da computação, topologia algébrica, lógica, categorias e álgebra superior, e um único conceito parece servir como um elo de ligação: "caminho". Seu impacto na matemática foi sentido com mais força desde o início do programa de V. Voevodsky sobre os fundamentos univalentes da matemática por volta de 2005, e um aspecto específco que gostaríamos de abordar aqui é o cálculo de grupos fundamentais de superfícies. Sabemos que o cálculo de grupos de homotopia é em geral muito mais difícil do que alguns dos outros invariantes de homotopia trabalhados em topologia algébrica. Agora, ao usar uma formulação alternativa do "tipo identidade"que fornece uma explicação formal explícita de "caminho", operacionalmente entendido como uma sequência inversível de reescritas (como a "conversão"de Church) e interpretado como uma homotopia, buscamos fornecer exemplos de cálculo de grupos fundamentais de superfícies, como o círculo, o toro, o 
toro de 2 furos, a garrafa de Klein e o plano projetivo real. Esses exemplos parecem testemunhar o impacto da MLTT na matemática, oferecendo ferramentas formais para calcular e provar grupos fundamentais, bem como permitir fazer tais cálculos e provas passíveis de serem tratadas por sistemas de matemática formalizada e provadores de teoremas interativos como Coq, Lean e outros semelhantes.

\section{Caminhos computacionais}

Dado um espaço $A$, podemos pensar numa estrutura $\Pi(A)$ formada por pontos do espaço $A$ e caminhos homotópicos entre esses pontos. Usando definições equacionais, pode-se facilmente provar para $\Pi(A)$ que as equações de grupóide valem a menos de homotopia. Isso é chamado de estrutura fraca (é fraca pois as igualdades não valem literalmente, mas a menos de homotopia): o grupóide fundamental do espaço $A$. Como para cada tipo $A$, usando a interpretação homotópica, pode ser semanticamente interpretado como sendo um espaço, podemos considerar $\Pi(A)$ como o grupóide fundamental do tipo $A$.

Como já mencionado anteriormente, os caminhos da interpretação homotópica das diferentes formulações da teoria de tipos de Martin-Löf não têm contrapartida na sintaxe, mas existem apenas na semântica. Adicionamos à sintaxe termos especificamente definidos para representar os caminhos computacionais e ainda assim permanecemos em consonância com os princípios da interpretação de Curry-Howard, pois esses termos representam provas de igualdade [de Queiroz et al. 2016, Ramos et al. 2017, de Queiroz and de Oliveira 2014].

Vamos construir um grupóide fundamental para um tipo $A$ usando caminhos de reescrita para os termos representando caminhos, e ainda assim mantendo essencialmente a mesma semântica oferecida para a homotopy type theory. Como demonstração da utilidade desse aparato formal, conforme mencionado anteriormente, temos trabalhado em exemplos de cálculo de grupos fundamentais de superfícies, como o círculo, o toro, o toro de 2 furos, a garrafa de Klein e o plano projetivo real.

Conforme a literatura sobre o $\lambda$-cálculo, a teoria equacional de termos estabelece quando dois $\lambda$-termos são iguais. Como exemplo ilustrativo, dado que $(\lambda x . x) z \triangleright_{\beta} z$, consideramos que $(\lambda x . x) z={ }_{\beta \eta} z$ em razão da existência do caminho $\beta((\lambda x . x) z, z)$. Considerando a formulação do tipo $\Pi$ dos $\lambda$-termos:$$
[x: A]
$$$$
[x: A]
$$$$
\text { (B) } \frac{N: A \quad M: B}{(\lambda x \cdot M) N=M[N / x]: B[N / x]}
$$

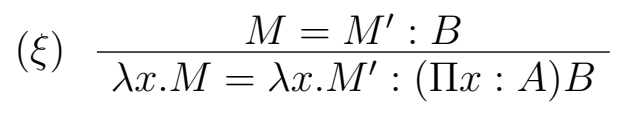$$
\text { ( } \rho) \frac{M: A}{M=M: A}
$$$$
\text { (u) } \frac{M=M^{\prime}: A \quad N:(\Pi x: A) B}{N M=N M^{\prime}: B[M / x]}
$$$$
\text { ( } \sigma) \frac{M=N: A}{N=M: A}
$$$$
\text { ( }) \frac{M=N: A \quad N=P: A}{M=P: A}
$$$$
(\nu) \frac{N: A \quad M=M^{\prime}:(\Pi x: A) B}{M N=M^{\prime} N: B[N / x]}
$$$$
\text { ( }) \frac{M:(\Pi x: A) B}{(\lambda x . M x)=M:(\Pi x: A) B}(x \notin F V(M))
$$

Definição 3.1 Sejam a e b elementos de um tipo A. Então, um caminho computacional $s$ de a para b é uma composição de aplicações das regras de inferência da teoria da igualdade da teoria de tipos. Denotamos por $a={ }_{s} b$. 
A composição é dada pela aplicação da regra da transitividade. A título de ilustração, considere a construção do caminho entre $(\lambda y . y x)(\lambda w . z w)$ e $z x$. Começando em $(\lambda y . y x)(\lambda w . z w)$, temos que $(\lambda y . y x)(\lambda w . z w) \triangleright_{\eta}(\lambda y . y x) z$. Daí, o caminho $\eta((\lambda y . y x)(\lambda w \cdot z w),(\lambda y \cdot y x) z)$. De $(\lambda y \cdot y x) z$, temos que $(\lambda y \cdot y x) z \triangleright_{\beta} z x$. Portanto, temos um caminho $\beta((\lambda y . y x) z, z x)$. Agora precisamos compor esses caminhos. Para isso, podemos aplicar a transitividade para obter o caminho desejado entre $(\lambda y . y x)(\lambda w . z w)$ e $z x$. Logo, a igualdade é estabelecida pelo caminho $\tau(\eta((\lambda y . y x)(\lambda w . z w),(\lambda y . y x) z), \beta((\lambda y . y x) z, z x))$. Portanto, um caminho computacional é um termo construído pela composição de identificadores de igualdades (definicionais). Tal qual a noção de redução entre termos que representam elementos de um tipo, é preciso definir as regras de redução para termos que representam caminhos, de forma que se obtenha a forma canônica de um caminho computacional. Nesse sentido, formulamos em [de Queiroz et al. 2011], um teorema da forma normal para caminhos computacionais, analisando as redundâncias em provas de igualdade, construindo regras de redução, e demonstrando terminação e confluência do sistema de reescrita associado.

\section{Reduções entre caminhos computacionais}

Considere o caso simples de um caminho reflexivo $\rho$ que estabelece que $a={ }_{\rho} a$. Se aplicarmos o axioma da simetria $\sigma$, obtemos o caminho $a={ }_{\sigma(\rho)} a$. Como aplicamos o axioma $\sigma$ ao caso trivial da reflexividade, obtemos um caminho que é uma forma redundante da reflexividade. Nesse caso, os caminhos $\rho$ e $\sigma(\rho)$ deveriam ser considerados iguais, i.e., deve ser possível reduzir $\sigma(\rho)$ a simplesmente $\rho$. O mesmo ocorre se, dado qualquer caminho $a={ }_{s} b$, aplicamos a simetria duas vezes em sequência, obtendo o caminho $a={ }_{\sigma(\sigma(s))} b$. Inverter um caminho duas vezes é equivalente a simplesmente usar o caminho original, i.e., $\sigma(\sigma(s))$ deveria ser reduzido a $s$.

As redundâncias mencionadas acima tiveram origem na combinação de aplicações dos axiomas da teoria da igualdade para a teoria de tipos, ou melhor, a partir da análise de redundâncias em provas construídas no estilo Dedução Natural decoradas com termos, tal qual na Curry-Howard. Na seção anterior, usando o exemplo do tipo produto $\Pi$, mostramos que temos um total de 8 regras/axiomas de igualdade. Dado que se pode combinar essas regras/axiomas em diversas situações, é de se esperar que o número de redundâncias distintas seja alto. Felizmente tais redundâncias foram identificadas e analisadas por [de Oliveira 1995], refinadas e associadas a identificadores em [de Queiroz et al. 2016]. Dessa forma, [de Oliveira 1995] criou um sistema que mapeia essas reduções, identificado como $L N D_{E Q}-T R S$, com um total de 39 regras. Para construir o grupóide fundamental, 7 regras serão essenciais:

Reduções envolvendo $\sigma$ e $\rho: \sigma(\rho) \triangleright_{s r} \rho \quad \sigma(\sigma(r)) \triangleright_{s s} r$

Reduções envolvendo $\tau: \tau(r, \sigma(r)) \triangleright_{t r} \rho \quad \tau(\sigma(r), r) \triangleright_{t s r} \rho \quad \tau(r, \rho) \triangleright_{t r r} r \quad \tau(\rho, r) \triangleright_{t l r} r$ Reduções envolvendo $\tau$ e $\tau: \tau(\tau(t, r), s) \triangleright_{t t} \tau(t, \tau(r, s))$

Como se pode ver, um identificador é associado a cada regra de reducção. Por exemplo, nossos exemplos iniciais são resolvidos por aplicações da regra $s r$ e a regra $s s$ respectivamente. É importante notar que o sistema $L N D_{E Q}-$ $T R S$ tem as propriedades de terminação e de confluência, como demonstrado em [de Oliveira 1995, de Queiroz and de Oliveira 1994, de Oliveira and de Queiroz 1999, de Queiroz et al. 2011]. Chamamos cada regra de redução de regra de reescrita e abreviamos por $r w$-regra. Associada a uma $r w$-regra, temos as seguintes definições: 
Definição 4.1 Sejam s e $t$ caminhos computacionais. Dizemos que $s \triangleright_{1 \text { rw }} t$ (leia-se: a rw-contrai para b) se pudermos obter $t$ a partir de s por uma aplicação de apenas uma rw-regra. Se s puder ser reduzido a t por um número finito de rw-contrações, então dizemos que $s \triangleright_{r w} t$ (leia-se s rw-reduz a t).

Definição 4.2 Sejam s e $t$ caminhos computacionais. Dizemos que $s={ }_{r w} t$ (leia-se: $s$ é rw-igual a t) sse t pode ser obtido de s por uma série finita (talvez vazia) de rwcontrações e rw-contrações reversas. Em outras palavras, $s={ }_{r w} t$ sse existe uma sequência $R_{0}, \ldots ., R_{n}$, com $n \geq 0$, tal que $(\forall i \leq n-1)\left(R_{i} \triangleright_{1 r w} R_{i+1}\right.$ ou $\left.R_{i+1} \triangleright_{1 r w} R_{i}\right)$ $\left(R_{0} \equiv s, \quad R_{n} \equiv t\right)$.

Por definição, a $r w$-igualdade é uma relação de equivalência, pois foi definida como o fecho reflexivo, simétrico e transitivo da $r w$-redução. Como uma $r w$-igualdade é dada por uma sequencia de $r w$-reduções, chamamos essa sequencia de $r w$-seqüência

\section{O grupóide fundamental de um tipo: $\Pi(A)$}

A seguir, vamos mostrar que a introdução de termos para caminhos traz benefícios, em particular, para a construção do grupóide fundamental de um tipo $A$. Inicialmente, é preciso se dar conta da ideia de que igualdade pode valer em um sentido fraco. Na interpretação homotópica a qualificação 'fraca' se aplica à estrutura para a qual as igualdades valem apenas a menos de homotopia. Usaremos o termo 'fraca' para caracterizar uma estrutura cujas igualdades valem apenas a menos de $r w$-igualdade. Se as igualdades valem literalmente, ao invés de serem fracas, dizemos que a estrutura é estrita. Primeiramente, vamos retomar alguns conceitos da teoria das categorias [Awodey 2010]:

Definição 5.1 Seja $f: A \rightarrow B$ uma seta de uma categoria qualquer. $f$ é dita ser um isomorfismo se existe uma $g: B \rightarrow$ A tal que $g \circ f=1_{A}$ e $f \circ g=1_{B}$. gé chamada de inversa de $f$ e pode ser escrita $f^{-1}$.

Definição 5.2 Um groupóide é uma categoria na qual toda seta é um isomorfismo.

Podemos pensar numa estrutura $\Pi(A)$ na qual os objetos são elementos $a: A$ e os morfismos são os caminhos computacionais entre esses objetos:

Proposição $5.1 \Pi(A)$ é um groupóide fraco.

Prova Primeiramente, é preciso provar que $\Pi(A)$ é uma categoria fraca. Para fazer isso, precisamos definir composição de morfismos e a seta identidade. Já sabemos que composição de caminhos $s \circ t$ é dada pela aplicação da transitividade, i.e., $s \circ t=\tau(t, s)$. $\mathrm{O}$ morfismo identidade de um objeto $a$ é dado pelo caminho reflexivo $a={ }_{\rho_{a}} a$. Agora é preciso verificar as leis de associatividade e identidade. A igualdade da associatividade vale fracamente, só precisamos usar a regra $t t: \tau(\tau(s, r), t)={ }_{r w_{t t}} \tau(s, \tau(r, t))$.

Usando as regras $t$ lr e $\operatorname{tr} r$, mostramos que as leis de identidade valem fracamente: $s \circ 1_{a}=$ $s \circ \rho_{a}=\tau\left(\rho_{a}, s\right)={ }_{r w_{t l r}} s$ e $1_{b} \circ s=\rho_{b} \circ s=\tau\left(s, \rho_{b}\right)={ }_{r w_{t r r}} s$.

Com essas condições satisfeitas, concluímos que $\Pi(A)$ é de fato uma categoria fraca. Agora precisamos mostrar que é um grupóide fraco. Para isso, precisamos mostrar que todo caminho computacional $s$ tem um inverso $s^{\prime}$. Encontrar $s^{\prime}$ é fácil, faça $s^{\prime}=\sigma(s)$. Para mostrar que as igualdades do isomorfismo valem fracamente, usamos as regras tr e $t s r: s \circ s^{\prime}=s \circ \sigma(s)=\tau(\sigma(s), s)={ }_{r w_{t s r}} \rho_{b}$ e $s^{\prime} \circ s=\sigma(s) \circ s=\tau(s, \sigma(s))=_{r w_{t r}} \rho_{a}$. 

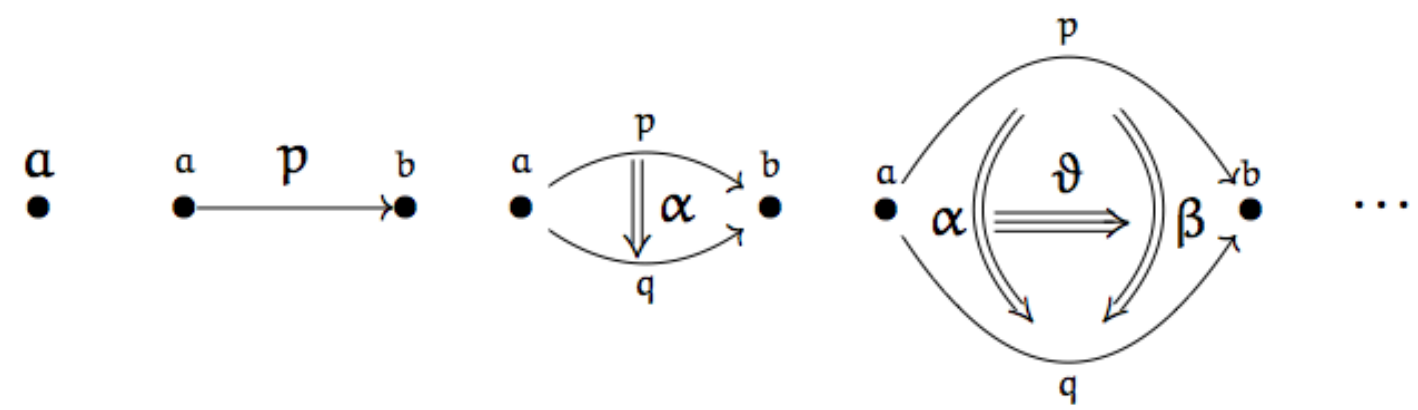

Figura 1. [Awodey 2017]

Como o grupóide $\Pi(A)$ é construído a partir de um tipo $A$ e seus objetos e igualdades entre esses objetos, definimos $\Pi(A)$ como o grupóide fundamental do tipo $A$. Como provado por [Hofmann and Streicher 1994], o modelo de groupóide de um tipo prova que a unicidade de provas de identidade não é derivável na sintaxe da teoria de tipos. A construção do grupóide fundamental de um tipo baseada em caminhos computacionais deixa claro que a incorporação à sintaxe de termos para caminhos ainda assim refuta a unicidade de provas de identidade. Portanto, pode-se interpretar esse resultado como dizendo que pode haver dois caminhos computacionais $s$ e $t$ entre objetos $a: A$ e $b: A$ tal que $s$ e $t$ não são $r w$-iguais.

O grupóide fundamental é um conceito fundamental que abre caminho para múltiplas linhas de pesquisa. A primeira possibilidade é usar o grupóide fundamental para obter grupóides de ordens superiores. Para isso, basta pensar que da mesma forma que existem caminhos entre dois tipos, também existem caminhos entre caminhos, os quais funcionam como uma prova da igualdade entre dois caminhos. Dessa forma, podemos considerar uma estrutura que para cada par de pontos $(a, b) \in \Pi(A)$, existe uma categoria fraca formada pelos caminhos entre os caminhos que conectam $a$ e $b$. Essa estrutura seria o grupóide fundamental de segunda ordem do tipo $A, \Pi_{2}(A)$. Provar que essa estrutura é realmente um grupóide de segunda ordem fica fora do escopo deste trabalho, mas a prova completa pode ser vista em [Ramos et al. 2017]. A linha de raciocínio envolvida nesse tipo de trabalho é ilustrada por [Awodey 2012, Awodey 2017] na exposição das conexões entre teoria dos tipos e teoria da homotopia (see Fig. 1):

$$
\begin{aligned}
\text { Tipos } & \rightsquigarrow \text { Espaços } \\
\text { Termos } & \rightsquigarrow \text { Mapas } \\
a: A & \rightsquigarrow \text { Pontos } a: 1 \rightarrow A \\
p: I d_{A}(a, b) & \rightsquigarrow \text { Caminhos } p: a \Rightarrow b \\
\alpha: I d_{I d_{A}(a, b)}(p, q) & \rightsquigarrow \text { Homotopias } \alpha: p \Rightarrow q \\
\vartheta: I d_{I d_{I d_{A}(a, b)}(p, q)}(\alpha, \beta) & \rightsquigarrow \text { Homotopias entre Homotopias } \vartheta: \alpha \Rightarrow \beta
\end{aligned}
$$

Uma segunda importante linha de pesquisa em que estamos desenvolvendo recentemente é a utilização do grupóide fundamental e caminhos computacionais para calcular os grupos fundamentais de superfícies. O primeiro resultado interessante obtido nesse sentido foi o cálculo que o grupo fundamental do círculo $S^{1}$ é equivalente aos inteiros $\mathbb{Z}$ 
[Ramos et al. 2018], tal qual o que foi obtido no livro Homotopy Type Theory ${ }^{2}$, mas dessa vez usando caminhos computacionais explícitos. Mais recentemente, também usamos caminhos computacionais para calcular o toro, o cilindro, a garrafa de Klein e o plano projetivo real [de Veras et al. 2019].

\section{Referências}

Awodey, S. (2010). Category theory. Oxford University Press.

Awodey, S. (2012). Type theory and homotopy. In Dybjer, P., Lindström, S., Palmgren, E., and Sundholm, G., editors, Epistemology versus Ontology, pages 183-201. Springer.

Awodey, S. (2017). A proposition is the (homotopy) type of its proofs. arXiv:1701.02024.

de Oliveira, A. G. (1995). Proof transformations for labelled natural deduction via term rewriting. Master's thesis. Depto. Informática, UFPE, Recife, Brazil, April 1995.

de Oliveira, A. G. and de Queiroz, R. J. G. B. (1999). A normalization procedure for the equational fragment of labelled natural deduction. Logic J. of IGPL, 7(2):173-215.

de Queiroz, R. J. G. B. and de Oliveira, A. G. (1994). Term rewriting systems with labelled deductive systems. In Proc. Brazilian Symp. on Artif. Intellig., pages 59-72.

de Queiroz, R. J. G. B. and de Oliveira, A. G. (2014). Natural deduction for equality: The missing entity. In Pereira, L. C., Haeusler, E., and de Paiva, V., editors, Advances in Natural Deduction - A Celebration of Dag Prawitz's Work, pages 63-91. Springer.

de Queiroz, R. J. G. B., de Oliveira, A. G., and Gabbay, D. M. (2011). The Functional Interpretation of Logical Deduction. World Scientific.

de Queiroz, R. J. G. B., de Oliveira, A. G., and Ramos, A. F. (2016). Propositional equality, identity types, and direct computational paths. South Am.J.Log., 2:245-296.

de Veras, T. M. L., Ramos, A. F., de Queiroz, R. J. G. B., and de Oliveira, A. G. (2019). A topological application of labelled natural deduction. arXiv 1906.09105.

Hindley, J. R. and Seldin, J. P. (2008). Lambda-calculus and combinators: an introduction. Cambridge University Press.

Hofmann, M. and Streicher, T. (1994). The groupoid model refutes uniqueness of identity proofs. In $L I C S^{\prime}$ '94, pages 208-212. IEEE.

Martínez-Rivillas, D. O. and de Queiroz, R. J. G. B. (2021). The $\infty$-groupoid generated by an arbitrary topological $\lambda$-model. Logic Journal of IGPL.

Ramos, A. F., de Queiroz, R. J. G. B., and de Oliveira, A. G. (2017). On the identity type as the type of computational paths. Logic Journal of the IGPL, 25(4):562-584.

Ramos, A. F., de Queiroz, R. J. G. B., de Oliveira, A. G., and de Veras, T. M. L. (2018). Explicit computational paths. South American Journal of Logic, 4(2):441-484.

Voevodsky, V. (2011). Univalent foundations of mathematics. In Beklemishev, L. D. and de Queiroz, R., editors, WoLLIC 2011, volume 6642 of LNAI, page 4. Springer.

\footnotetext{
${ }^{2}$ https://homotopytypetheory.org/book
} 